\title{
Datiloscopia e APTIDÃo físICA dos INTEGRANTES do Centro de Iniciação e Especialização de Atletismo da Primeira Região
}

\author{
Jorge Díaz'jediaz@uta.cl \\ Omar Espinoza' oespinoz@uta.cl
}

doi: 10.3900/fpi.7.4.209.p

Díaz J, Espinoza O. Datiloscopia e aptidão física dos integrantes do Centro de Iniciação e Especialização de Atletismo da Primeira Região. Fit Perf J. 2008 jul-ago;7(4):209-16.

\begin{abstract}
RESUMO
Introdução: A presente pesquisa teve como finalidade identificar a relação existente entre os desenhos dactiloscópicos e a aptidão física nos integrantes do Centro de Iniciação e Especialização Esportiva de Atletismo da Primeira Região. Materiais e Métodos: A amostra foi composta por 14 mulheres e 15 homens, com idades de 12 a 17 anos. Os sujeitos selecionados para o estudo foram submetidos a uma bateria de testes para determinar o perfil genético e a aptidão física. $O$ perfil genético foi medido pelo método proposto por Abramova et al. (1995). A aptidão física foi medida através da aplicação dos seguintes testes: a) velocidade - Teste de $50 \mathrm{~m}$; b) potência aeróbica - Teste de 6 min de Corrida Contínua; c) força explosiva: Teste de Sargent; d) agilidade: Teste de Ida e Volta (drible). No tratamento e análise dos dados foram utilizados estadígrafos de tendência central, de dispersão, grau de significância a $p<0,005$ e correlação. Resultados: Os resultados mostram uma grande associação entre a aptidão física e o perfil genético. Discussão: Destaca-se uma alta correlação das impressões digitais L-A com as atletas de velocidade e lançadoras. Por sua vez, o design $W$ em combinação com $L$ se associa com os atletas fundistas.
\end{abstract}

\section{PALAVRAS-CHAVE}

Dermatoglifia, Aptidão Física, Esportes.

${ }^{1}$ Universidad de Tarapacá - Arica - Chile

Copyright@ 2008 por Colégio Brasileiro de Atividade Física, Saúde e Esporte

Fit Perf J | Rio de Janeiro | 7 | 4 | 209-16 | jul/ago 2008 
Fingerprint identification and physical aptitude of integrants of the Center of Initiation and Specialization of Athletics in the First Region

\begin{abstract}
Introduction: The present research aimed at identifying the relation between the fingerprint data and the physical aptitude in the integrants of the Center of Athletics Sport Initiation and Specialization in the First Region. Materials and Methods: The sample was composed of 14 women and 15 men, aged between 12 and 17 years old. The individuals selected for the study were submitted to an amount of tests to determine the genetic profile and the physical aptitude. The genetic profile was measured with the method proposed by Abramova et al. (1995). The physical aptitude was measured by the application of the following tests: a) velocity - $50 \mathrm{~m}$ Test; b) aerobic potency - 6min Continuous Run Test; c) explosive strength - Sargent Test; d) agility: Two Way Test (dribble). In the treatment and analysis of the data we used: stadigraphs of central tendency, dispersion, significance degree to $p<0,005$ and correlation. Results: The results showed a great association between the physical aptitude and the genetic profile. Discussion: a high correlation between the fingerprints L-A and velocity athletes and throwers is highlighted. On the other hand, the design W in combination with $L$ is associated to long distance runners.
\end{abstract}

\title{
KEYWORDS
}

Dermatoglyphics, Physical Fitness, Sports.

\section{Datiloscopia y aptitud física de los integrantes del Centro de Iniciación y Especialización de Atletismo de la Primera Región}

\section{RESUMEN}

Introducción: La presente investigación tuvo como finalidad identificar la relación existente entre los dibujos dactiloscópicos y la aptitud física en los integrantes del Centro de Iniciación y Especialización Deportiva (CIED) de Atletismo de la Primera Región. Materiales y Métodos: La muestra fue compuesta por 14 mujeres y 15 hombres, con edades de 12 a 17 años. Los sujetos seleccionados para el estudio habían sido sometidos a una batería de tests para determinar el perfil genético y la aptitud física. El perfil genético fue medido por el método propuesto por Abramova et al. (1995). La aptitud física fue medida a través de la aplicación de los siguientes tests: a) velocidad - Test de 50m; b) potencia aeróbica - Test de 6 min de Carrera Continua; c) fuerza explosiva: Test de Sargent; d) agilidad: Test de Ida y Vuelta (regate). En el tratamiento y análisis de los datos habían sido utilizados estadígrafos de tendencia central, de dispersión, grado de acepción a $p<0,005$ y correlación. Resultados: Los resultados muestran una gran asociación entre la aptitud física y lo perfil genético. Discusión: Se destaca un alza correlación de las huellas L-A con las atletas de velocidad y lanzadoras. A su vez, el diseño $W$ en combinación con $L$ se asocia con los atletas fondistas.

\section{PALABRAS CLAVE}

Dermatoglifia, Acondicionamiento Físico, Deportes.

\section{INTRODUÇÃO}

Atualmente, os altos rendimentos alcançados pelas grandes potências esportivas do mundo baseiam estes resultados na busca científica de jovens talentos que sejam capazes de receber grandes cargas de treinamento e elevados ritmos de aperfeiçoamento esportivo. Isto se confirma pelo que foi dito por Filin \& Volkov ${ }^{1}$, que afirmam que a seleção esportiva é produzida em períodos e em cada uma delas existem tarefas e orientações específicas. É assim com a escola soviética, onde a detecção de talentos esportivos é realizada a partir de pesquisas com crianças, por meio de métodos pedagógicos, morfológicos, psicológicos e genéticos. $\bigcirc$ objetivo desta orientação e seleção esportiva é a descoberta de sujeitos com talento, obtendo a definição dos potenciais genéticos esportivos que possuem para a obtenção do alto rendimento.

Conhecimento do potencial genético permite diferenciar os componentes físicos, os aspectos fracos e fortes, o prognóstico e as possibilidades da criança no seu desenvolvimento esportivo. Isto permite assegurar o aperfeiçoamento ativo dos componentes fortes com uma preparação adequada. Da mesma forma, também permite processar o desenvolvimento dos pontos fracos pouco favorecidos pelo potencial genético.

Na atualidade, a dermatoglifia é um método simples para a determinação das capacidades e possibilidades dos atletas jovens ${ }^{2}$. É considerada como uma ciência que estuda os relevos e desenhos da ponta dos dedos, da palma das mãos e da planta dos pés.

Neste âmbito, a maioria dos autores distingue três tipos de desenhos: Arco (A), ou desenho sem delta; Presilha (L), ou desenho de um delta; Verticilo (W), ou desenho de dois deltas. A forma dos desenhos constitui uma característica qualitativa do ser humano (D10) e a somatória total de linhas (SQTL) representam as características quantitativas. 
Desta maneira, as impressões digitais são estruturas hereditariamente determinadas que possuem uma multiformidade estrutural por serem diferenciadas filogenética e antropogeneticamente para a execução das funções mecânicas e táteis complexas, que se distinguem por sua incomparabilidade individual; são marcas genéticas universais.

A este respeito, Fernandes Filho² afirma que, na década de 60, iniciou-se na URSS uma série de estudos destinados a otimizar o desenvolvimento esportivo de sua juventude. Entre estes, surgem os trabalhos realizados por Bulgakova $^{3}$, Nikitiuk \& Filipov ${ }^{4}$, Abramova \& Nikitina $^{5}$, Abramova et al. ${ }^{6,7}$, e outros cientistas que confirmam a aceitabilidade das impressões digitais (ID) como uma fonte de informação genética que permite determinar as potencialidades de desenvolvimento e rendimento físico de um sujeito. Os estudos realizados pelos soviéticos permitiram relacionar, com um alto índice de significância $(p<0,001)$, as possibilidades do rendimento esportivo e as ID. Com relação a isto, Abramova et al. ${ }^{6}$ afirmam que a interação de fatores em comum entre as ID e a modalidade esportiva reflete a lei biológica natural da inter-relação das marcas genéticas com a aptidão congênita (dotes), das manifestações funcionais, independente da pertinência da população. Por conseguinte, as ID podem ser utilizadas como critério de orientação esportiva precoce e de seleção esportiva da população infantil e adolescente de qualquer país.

Atualmente, no Chile, existem 13 Centros de Iniciação e Especialização Esportiva (CIED), integrados por diferentes esportes (os mais desenvolvidos ou destacados de cada região). Estes CIED têm como finalidade a captação e o desenvolvimento dos talentos esportivos de cada região. No entanto, apesar desta política de desenvolvimento implementada por Digeder e continuada na atualidade pelo Chile Esporte, não existe um desenho ov estratégia técnica que permita identificar os aspectos mais relevantes de um talento esportivo, como os da composição corporal, somatótipo, aptidão física e perfil genético das crianças e adolescentes que participam deles. Por tal razão, achamos que a aplicação piloto de um estudo desta natureza, em uma população escolhida, poderia reafirmar a capacidade e experiência dos técnicos e treinadores na detecção, captação e desenvolvimento do talento esportivo, e, se assim não fora, esta investigação permitirá contribuir para a informação genética e composição corporal deste recurso esportivo.

\section{Objetivo geral}

Identificar a relação existente entre os desenhos dactiloscópicos e a aptidão física nos integrantes do CIED de Atletismo da Primeira Região.

\section{Objetivos específicos}

- Identificar as características genéticas (dactilogramas), dos integrantes do CIED de Atletismo da Primeira Região;

- Identificar o nível de desenvolvimento da aptidão física dos integrantes do CIED de Atletismo da Primeira Região.

\section{Hipóteses}

HO - Não existe correlação significativa entre as características dermatoglíficas e a aptidão física dos atletas integrantes do CIED da Primeira Região.

$\mathrm{H} 1$ - Existe correlação significativa entre as características dermatoglíficas e a aptidão física dos atletas integrantes do CIED da Primeira Região.

\section{Variáveis independentes}

- Perfil genético (tipo de desempenho das impressões digitais, D10 e SQTL);

- Perfil de aptidão física (potência aeróbica, agilidade, velocidade, força explosiva).

\section{Variável dependente}

- Idade, sexo, atletas integrantes do CIED da Primeira Região.

\section{MATERIAIS E MÉTODOS}

$\bigcirc$ presente estudo corresponde a uma pesquisa de tipo descritiva comparativa e correlacional. A amostra foi composta por 29 sujeitos (14 mulheres e 15 homens), com idades entre 12 e 17 anos, integrantes do CIED. Os sujeitos selecionados para o estudo foram submetidos a uma

Tabela 1 - Classificação dermatoglífica das mulheres integrantes do CIED de Atletismo da I Região

\begin{tabular}{llcccc}
\hline \multirow{2}{*}{ Classe } & $\mathrm{n}$ & \multicolumn{2}{c}{ impressões digitais } & mínimo & máximo \\
\cline { 3 - 6 } & 4 & D10 & SQTL & estatura força resistência coordenação & força relativa \\
I & 5,75 & 29,5 & coordenação & força \\
II & 4 & 10,25 & 99,5 & força relativa & estatura - força absoluta \\
III & 3 & 12,0 & 91,33 & estatura força absoluta & resistência coordenação \\
IV & 3 & 13,7 & 79,21 &
\end{tabular}


bateria de testes para determinar: a) perfil genético; e b) aptidão física. $\bigcirc$ perfil genético foi determinado mediante o método proposto por Abramova et al. 6 . A aptidão física foi avaliada mediante a aplicação dos seguintes testes: velocidade - Teste de 50 m; potência aeróbica - Teste de 6min de Corrida Contínua; força explosiva - Teste de Sargent (saltar e alcançar); agilidade: Teste de Ida e Volta (drible). O protocolo utilizado para a coleta de dados foi o Dermatoglífico ${ }^{8}$. Este método se inicia com a obtenção das ID e seu processamento posterior. A impressão das ID de todos os dedos se realizou em uma ficha confeccionada especialmente para isso. Depois da obtenção das ID se realizou o processamento preliminar destas, para o qual se utilizou o padrão proposto por Cummins \& Midlo, citado por Fernandes Filho ${ }^{2}$. No tratamento e análise dos dados se utilizaram estadígrafos de tendência central, de dispersão, grau de significância $p<0,005$ e correlação.

\section{RESULTADOS}

\section{Características dermatoglíficas}

Os dados obtidos da aplicação dos testes dermatoglíficos permitiram caracterizar a amostra em termo das relações possíveis entre as potencialidades físicas e as ID. Assim, na Tabela 1 se apresenta a classificação das características dermatoglíficas das mulheres, na qual se pode dizer que $28,6 \%$ delas se encontram localizadas no Nível I, o que significa que as quatro apresentam bom desenvolvimento da força relativa, isto é, da relação força/peso corporal. Além disso, se observa que, no fator somático, a estatura, a força de resistência e a coordenação são qualidades fracas geneticamente, que necessitam atenção e estimulação para seu desenvolvimento.

No que diz respeito ao grupo classificado no Nível II, pode-se afirmar que $28,6 \%$ das atletas em estudo apresentam como característica fundamental a força, isto é, são sujeitos que possuem um bom desenvolvimento muscular, mas com uma coordenação de baixo desenvolvimento. No Nível III se encontra 21,4\% dos sujeitos em estudo, que apresentam como ponto forte um bom desenvolvimento da estatura e sua força absoluta se encontra com um grande potencial de desenvolvimento. No entanto, a força relativa se mostra como uma debilidade, o que indica a necessidade de se preocupar em estimular seu desenvolvimento. Logo, ao grupo situado no Nível IV, equivalente a $21,4 \%$, sua prevalência genética se encon-

Tabela 2 - Classificação dermatoglífica dos homens integrantes do CIED de Atletismo da I Região

\begin{tabular}{|c|c|c|c|c|c|}
\hline \multirow{2}{*}{ classe } & \multirow{2}{*}{$\mathrm{n}$} & \multicolumn{2}{|c|}{ impressões digitais } & \multicolumn{2}{|c|}{ somáticos - funcionais } \\
\hline & & $\mathrm{D} 10$ & SQTL & mínimo & máximo \\
\hline I & 1 & 1 & 0 & estatura força resistência coordenação & força relativa \\
\hline ॥ & 4 & 10 & 80,75 & coordenação & força \\
\hline III & 3 & 12 & 70,33 & força relativa & estatura - força absoluta \\
\hline IV & 3 & 14,33 & 130,66 & estatura força absoluta & resistência coordenação \\
\hline V & & & & força relativa & coordenação \\
\hline
\end{tabular}

Tabela 3 - Quantidade de linhas dos dedos das mãos das mulheres velocistas

\begin{tabular}{ccccccccccccc}
\hline \multicolumn{10}{c}{ Mulheres Velocistas } \\
\hline \multicolumn{10}{c}{} & \multicolumn{10}{c}{ mão direita } & \multicolumn{1}{c}{ mão esquerda } \\
\hline $\mathrm{n}^{\circ}$ & $\mathrm{P}$ & $\mathrm{I}$ & $\mathrm{M}$ & $\mathrm{AN}$ & $\mathrm{MN}$ & $\mathrm{SQTL}$ & $\mathrm{P}$ & $\mathrm{I} N$ & $\mathrm{M}$ & $\mathrm{AN}$ & $\mathrm{MN}$ & $\mathrm{SQTL}$ \\
1 & $10 \mathrm{~L}$ & $3 \mathrm{~L}$ & $6 \mathrm{~L}$ & $6 \mathrm{~L}$ & $6 \mathrm{~L}$ & 31 & $6 \mathrm{~L}$ & $4 \mathrm{~L}$ & $6 \mathrm{~L}$ & $6 \mathrm{~L}$ & $4 \mathrm{~L}$ & 26 \\
2 & $12 \mathrm{~L}$ & $\mathrm{~A}$ & $5 \mathrm{~L}$ & $7 \mathrm{~L}$ & $5 \mathrm{~L}$ & 26 & $9 \mathrm{~L}$ & $11 \mathrm{~L}$ & $10 \mathrm{~L}$ & $\mathrm{~A}$ & $5 \mathrm{~L}$ & 25 \\
3 & $15 \mathrm{~L}$ & $3,5 \mathrm{~W}$ & $\mathrm{~A}$ & $13 \mathrm{~L}$ & $10 \mathrm{~L}$ & 41,5 & $6,5 \mathrm{~W}$ & $\mathrm{~A}$ & $4 \mathrm{~L}$ & $2 \mathrm{~L}$ & $8 \mathrm{~L}$ & 20,5 \\
4 & $14,4 \mathrm{~W}$ & $17,5 \mathrm{~W}$ & $10 \mathrm{~W}$ & $19,5 \mathrm{~W}$ & $11 \mathrm{~L}$ & 72,5 & $11 \mathrm{~W}$ & $14 \mathrm{~W}$ & $13 \mathrm{~L}$ & $16 \mathrm{~L}$ & $13 \mathrm{~L}$ & 67 \\
5 & $5 \mathrm{~L}$ & $1 \mathrm{~A}$ & $1 \mathrm{~A}$ & $4 \mathrm{~L}$ & $1 \mathrm{~A}$ & 9 & $2 \mathrm{~L}$ & $1 \mathrm{~A}$ & $1 \mathrm{~A}$ & $1 \mathrm{~A}$ & $1 \mathrm{~A}$ & 2 \\
$\Sigma$ & 56,4 & 30 & 21 & 49,5 & 32 & 180 & 34,5 & 30 & 33 & 24 & 30 & 150,5 \\
$\mathrm{x}$ & 11,28 & 6 & 5,25 & 9,9 & 8 & 36 & 6,9 & 7,25 & 8,25 & 6 & 7,5 & 30,1 \\
$\mathrm{dp}$ & 4,1 & 5,8 & 3,0 & 5,1 & 2,9 & 18,6 & 2,9 & 4,6 & 3,4 & 5,2 & 3,3 & 18,3 \\
\hline
\end{tabular}


Tabela 4 - Quantidade de linhas dos dedos das mãos das mulheres lançadoras e fundistas

\begin{tabular}{|c|c|c|c|c|c|c|c|c|c|c|c|c|}
\hline \multicolumn{13}{|c|}{ Mulheres Lançadoras } \\
\hline \multirow[b]{2}{*}{$n^{\circ}$} & \multicolumn{6}{|c|}{ mão direita } & \multicolumn{6}{|c|}{ mão esquerda } \\
\hline & $P$ & I & M & AN & $M M$ & SQTL & $P$ & IN & M & AN & $M M$ & SQTL \\
\hline 1 & $16 \mathrm{~W}$ & $9 \mathrm{~L}$ & $10 \mathrm{~L}$ & $14 \mathrm{~L}$ & $8 \mathrm{~L}$ & 57 & $10 \mathrm{~W}$ & $13 \mathrm{~L}$ & $17 \mathrm{~L}$ & $16 \mathrm{~W}$ & $1 \mathrm{~A}$ & 56 \\
\hline 2 & $7 \mathrm{~L}$ & $10 \mathrm{~W}$ & $11 \mathrm{~L}$ & $16 \mathrm{~W}$ & $16 \mathrm{~L}$ & 60 & $5 \mathrm{~L}$ & $7 \mathrm{~L}$ & $10 \mathrm{~L}$ & $17 \mathrm{~L}$ & $13 \mathrm{~L}$ & 52 \\
\hline 3 & $14 \mathrm{~W}$ & $18 \mathrm{~L}$ & $10 \mathrm{~L}$ & $14 \mathrm{~W}$ & $13 \mathrm{~L}$ & 69 & $16 \mathrm{~W}$ & $16 \mathrm{~L}$ & $15 \mathrm{~L}$ & $15 \mathrm{~L}$ & $1 \mathrm{~A}$ & 62 \\
\hline 4 & $14 \mathrm{~W}$ & $1 \mathrm{~A}$ & $10 \mathrm{~L}$ & $1 \mathrm{~A}$ & $3 \mathrm{~L}$ & 27 & $6 \mathrm{~W}$ & $8 \mathrm{~L}$ & $1 \mathrm{~A}$ & $1 \mathrm{~A}$ & $1 \mathrm{~A}$ & 14 \\
\hline$\Sigma$ & 51 & 37 & 41 & 44 & 40 & 213 & 82 & 54 & 42 & 48 & 35 & 265 \\
\hline$x$ & 12,75 & 12,3 & 10,25 & 14,67 & 10,67 & 53,25 & 20,5 & 13,5 & 14 & 16 & 13 & 66,25 \\
\hline$d p$ & 3,4 & 4,0 & 0,4 & 0,9 & 5,6 & 15,8 & 17,4 & 3,5 & 2,9 & 0,8 & 0,0 & 29,1 \\
\hline \multicolumn{13}{|c|}{ Mulheres Fundistas } \\
\hline & \multicolumn{6}{|c|}{ mão direita } & \multicolumn{6}{|c|}{ mão esquerda } \\
\hline $\mathrm{n}^{\circ}$ & $P$ & I & M & AN & MM & SQTL & $P$ & IN & M & AN & MM & SQTL \\
\hline 1 & $1 \mathrm{~A}$ & $1 \mathrm{~A}$ & $1 \mathrm{~A}$ & $1 \mathrm{~A}$ & $1 \mathrm{~A}$ & 0 & $1 \mathrm{~A}$ & $1 \mathrm{~A}$ & $1 \mathrm{~A}$ & $1 \mathrm{~A}$ & $1 \mathrm{~A}$ & 0 \\
\hline 2 & $17,5 \mathrm{~W}$ & $16 \mathrm{~W}$ & $18 \mathrm{~W}$ & $15 \mathrm{~W}$ & 13,5 & 13,5 & $20 \mathrm{~W}$ & $17 \mathrm{~W}$ & $18,5 \mathrm{~W}$ & $20,5 \mathrm{~W}$ & $15,5 \mathrm{~W}$ & 91 \\
\hline 3 & $8 \mathrm{~L}$ & $10 \mathrm{~L}$ & $12 \mathrm{~L}$ & $14 \mathrm{~L}$ & $8 W$ & 52 & $1 \mathrm{~A}$ & $1 \mathrm{~A}$ & $1 \mathrm{~A}$ & $12 \mathrm{~W}$ & $1 \mathrm{~A}$ & 20,5 \\
\hline 4 & $10 \mathrm{~W}$ & $11 \mathrm{~L}$ & $7 \mathrm{~L}$ & $12 \mathrm{~L}$ & $9 \mathrm{~L}$ & 49 & $12 \mathrm{~W}$ & $6 \mathrm{~L}$ & $5 \mathrm{~L}$ & $10 \mathrm{~W}$ & $9 \mathrm{~L}$ & 42 \\
\hline 5 & $14 \mathrm{~L}$ & $17 \mathrm{~L}$ & $12 \mathrm{~L}$ & $10 \mathrm{~W}$ & $1 \mathrm{~A}$ & 43 & $8 \mathrm{~L}$ & $12 \mathrm{~W}$ & $12 \mathrm{~W}$ & $11 \mathrm{~W}$ & $1 \mathrm{~A}$ & 43 \\
\hline$\Sigma$ & 49,5 & 54 & 49 & 51 & 30,5 & 234 & 40 & 35 & 35,5 & 64,5 & 24,5 & 196,5 \\
\hline$x$ & 12,37 & 13,5 & 12,25 & 12,75 & 11,25 & 56,5 & 13,33 & 11,67 & 11,83 & 15,38 & 12,25 & 49,13 \\
\hline$d p$ & 3,7 & 3,0 & 3,9 & 1,9 & 2,3 & 13,9 & 5,0 & 4,5 & 5,5 & 4,9 & 3,3 & 25,8 \\
\hline
\end{tabular}

Tabela 5 - Quantidade de linhas dos dedos das mãos dos homens fundistas

\begin{tabular}{cccccccccccccc}
\hline \multicolumn{10}{c}{ mão direita } & \multicolumn{10}{c}{ Homens Fundistas } \\
\hline $\mathrm{n}^{\circ}$ & $\mathrm{P}$ & $\mathrm{IN}$ & $\mathrm{M}$ & $\mathrm{AN}$ & $\mathrm{MN}$ & $\mathrm{SQTL}$ & $\mathrm{P}$ & $\mathrm{IN}$ & $\mathrm{M}$ & $\mathrm{AN}$ & $\mathrm{MN}$ & $\mathrm{SQTL}$ \\
1 & $10 \mathrm{~W}$ & $\mathrm{~A}$ & $\mathrm{~A}$ & $5 \mathrm{~W}$ & $7 \mathrm{~W}$ & 22 & $11 \mathrm{~W}$ & $2 \mathrm{~L}$ & $\mathrm{~A}$ & $\mathrm{~A}$ & $10 \mathrm{~L}$ & 23 \\
2 & $14 \mathrm{~W}$ & $\mathrm{~A}$ & $5 \mathrm{~L}$ & $8 \mathrm{~L}$ & $6 \mathrm{~L}$ & 32 & $18 \mathrm{~L}$ & $9 \mathrm{~L}$ & $10 \mathrm{~L}$ & $12 \mathrm{~L}$ & $14 \mathrm{~L}$ & 53 \\
3 & $10 \mathrm{~W}$ & $\mathrm{~A}$ & $10 \mathrm{~L}$ & $16 \mathrm{~L}$ & $16 \mathrm{~L}$ & 52 & $8 \mathrm{~W}$ & $\mathrm{~A}$ & $6 \mathrm{~L}$ & $11 \mathrm{~L}$ & $10 \mathrm{~L}$ & 35 \\
4 & $9 \mathrm{~L}$ & $11 \mathrm{~L}$ & $9 \mathrm{~L}$ & $2 \mathrm{~L}$ & $4 \mathrm{~L}$ & 35 & $9 \mathrm{~L}$ & $8 \mathrm{~L}$ & $8 \mathrm{~L}$ & $3 \mathrm{~L}$ & $2 \mathrm{~L}$ & 30 \\
5 & $12 \mathrm{~L}$ & $9 \mathrm{~L}$ & $5 \mathrm{~L}$ & $9 \mathrm{~L}$ & $4 \mathrm{~L}$ & 39 & $9 \mathrm{~L}$ & $8 \mathrm{~L}$ & $16 \mathrm{~L}$ & $3 \mathrm{~L}$ & $1 \mathrm{~L}$ & 37 \\
6 & $9 \mathrm{~L}$ & $7 \mathrm{~L}$ & $6 \mathrm{~L}$ & $11 \mathrm{~L}$ & $7 \mathrm{~L}$ & 40 & 0 & $6 \mathrm{~L}$ & $6 \mathrm{~L}$ & $11 \mathrm{~L}$ & $6 \mathrm{~L}$ & 29 \\
$\Sigma$ & 64 & 27 & 35 & 51 & 44 & 221 & 55 & 33 & 46 & 40 & 43 & 207 \\
$\mathrm{x}$ & 10,7 & 4,50 & 5,83 & 9,83 & 7,33 & 36,67 & 9,17 & 5,50 & 7,67 & 6,67 & 7,17 & 34,50 \\
$\mathrm{dp}$ & 1,8 & 4,6 & 3,2 & 5,2 & 4,1 & 9,0 & 5,3 & 3,4 & 4,8 & 4,8 & 4,6 & 9,4 \\
\hline
\end{tabular}


Tabela 6 - Quantidade de linhas dos dedos das mãos dos homens velocistas

\begin{tabular}{ccccccccccccc}
\hline \multicolumn{10}{c}{ mão direita } & \multicolumn{10}{c}{ Momens Velocistas } \\
\hline $\mathrm{n}^{\circ}$ & $\mathrm{P}$ & $\mathrm{IN}$ & $\mathrm{M}$ & $\mathrm{AN}$ & $\mathrm{MN}$ & $\mathrm{SQTL}$ & $\mathrm{P}$ & $\mathrm{IN}$ & $\mathrm{M}$ & $\mathrm{AN}$ & $\mathrm{MN}$ & $\mathrm{SQTL}$ \\
1 & $21 \mathrm{~W}$ & $13 \mathrm{~L}$ & $12 \mathrm{~L}$ & $16 \mathrm{~W}$ & $3 \mathrm{~L}$ & 65 & $21 \mathrm{~W}$ & $13 \mathrm{~L}$ & $15 \mathrm{~L}$ & $14,5 \mathrm{~W}$ & $15 \mathrm{~L}$ & 78,5 \\
2 & $11 \mathrm{~L}$ & $10 \mathrm{~W}$ & $8 \mathrm{~L}$ & $8 \mathrm{~L}$ & $7 \mathrm{~W}$ & 41 & $11 \mathrm{~L}$ & $12 \mathrm{~L}$ & $13 \mathrm{~L}$ & $12 \mathrm{~L}$ & $7 \mathrm{~L}$ & 55 \\
3 & $18 \mathrm{~W}$ & $13 \mathrm{~W}$ & $\mathrm{~L} 7$ & $12 \mathrm{~W}$ & $15 \mathrm{~L}$ & 65 & $17 \mathrm{~W}$ & $12 \mathrm{~W}$ & $12 \mathrm{~L}$ & $16 \mathrm{~L}$ & $17 \mathrm{~L}$ & 73 \\
4 & $18 \mathrm{~W}$ & $8 \mathrm{~W}$ & $7 \mathrm{~W}$ & $14 \mathrm{~L}$ & $14 \mathrm{~L}$ & 61 & $21 \mathrm{~L}$ & $9 \mathrm{~L}$ & $\mathrm{~A}$ & $9 \mathrm{~L}$ & $\mathrm{~A}$ & 39 \\
5 & $10,5 \mathrm{~W}$ & $13 \mathrm{~L}$ & $\mathrm{~A}$ & $16 \mathrm{~L}$ & $2 \mathrm{~L}$ & 41,5 & $2 \mathrm{~L}$ & $35 \mathrm{~W}$ & $1 \mathrm{~L}$ & $16 \mathrm{~L}$ & $7 \mathrm{~L}$ & 61 \\
6 & $\mathrm{~A}$ & $\mathrm{~A}$ & $\mathrm{~A}$ & $\mathrm{~A}$ & $\mathrm{~A}$ & 0 & $\mathrm{~A}$ & $\mathrm{~A}$ & $\mathrm{~A}$ & $\mathrm{~A}$ & $\mathrm{~A}$ & 0 \\
7 & $18 \mathrm{~L}$ & $9 \mathrm{~L}$ & $9 \mathrm{~L}$ & $12,5 \mathrm{~W}$ & $11 \mathrm{~L}$ & 59,5 & $10 \mathrm{~L}$ & $6 \mathrm{~L}$ & $7 \mathrm{~L}$ & $8 \mathrm{~L}$ & $9 \mathrm{~L}$ & 40 \\
$\Sigma$ & 96,5 & 66 & 43 & 78,5 & 52 & 333 & 82 & 87 & 48 & 75,5 & 55 & 346,5 \\
$\mathrm{x}$ & 11,5 & 8,80 & 4,40 & 10,00 & 7,60 & 41,70 & 10,2 & 13,6 & 5,20 & 10,60 & 6,20 & 45,60 \\
$\mathrm{dp}$ & 6,7 & 4,3 & 4,2 & 5,3 & 5,6 & 21,7 & 7,9 & 10,1 & 6,1 & 5,3 & 6,1 & 24,6 \\
\hline
\end{tabular}

tra em um bom nível de desenvolvimento da resistência e da coordenação.

No caso dos atletas homens, cujos dados se apresentam na Tabela 2, pode-se afirmar que suas características dermatoglíficas são diferentes do observado nas mulheres.

É assim que $59,99 \%$ se concentram nos níveis II e III, mostrando no Nível II como ponto forte a força e no Nível III a estatura e força absoluta, sendo como debilidades somáticas a coordenação e a força relativa. Da amostra, 20\% se encontra no Nível I, onde seu potencial genético é a força relativa e suas debilidades a estatura, a força de resistência e a coordenação. Este nível é o que apresenta o maior número de qualidades deficitárias ou de fraco desenvolvimento. Os 20\% restantes ficam no Nível IV, onde seu ponto forte genético é a resistência e a coordenação, em oposição às debilidades de estatura e força absoluta.

\section{Intensidade sumária dos desenhos dermatoglíficos}

Para a análise específica dos sujeitos em estudo, se agruparam de acordo com a especialidade atlética que praticam. Assim, nas mulheres se distingue três grupos, velocistas, lançadoras e fundistas, e, nos homens, velocistas e fundistas.

Na Tabela 3 se apresentam o detalhe da somatória, as linhas dos diferentes desenhos em cada um dos dedos das mãos. Neste sentido, a especificação aponta os valores médios de intensidade da síntese dos desenhos em cada dedo das mãos e como se distribuem os desenhos dermatoglíficos nos dedos das mãos. Aqui se pode afirmar que, no caso das velocistas, a maior percentagem de linhas apresenta-se no polegar da mão direita, com relação ao resto dos dedos da mão. Entretanto, no grupo de lançadoras, esta situação é mais relativa, isto é, as médias de linhas por dedo são muito similares. No entanto, se observa na sua mão esquerda uma maior somatória em todos seus dedos, chegando a um SQTL de 66,25 de média, o que marca uma diferença de $13 \%$ em relação à mão direita.

Vale destacar que os sujeitos 1 e 2 são corredoras de 12,8 a 12,6 nos $100 \mathrm{~m}$ rasos. Além disso, a segunda tem recorde nacional de salto triplo categoria intermediária.

Com relação ao grupo de fundistas podemos manifestar que, neste caso, da mesma forma que nas velocistas, são os dedos da mão direita que apresentam o maior somatório de linhas refletindo isto em um maior SQTL. Em geral, pode-se dizer que o desenho de tipo $L$ é o de maior freqüência.

No que diz respeito aos homens, os dados apresentados na Tabela 5 confirmam o fato de que estes possuem maior intensidade de síntese (mão direita e esquerda)

Tabela 7 - Média e desvio padrão dos índices dermatoglíficos das mulheres e homens, segundo a especialidade atlética que praticam

\begin{tabular}{|c|c|c|c|c|c|c|c|}
\hline especialidade & $\mathrm{n}$ & & L & W & $A$ & D 10 & SQTL \\
\hline \multicolumn{8}{|c|}{ Mulheres } \\
\hline \multirow{2}{*}{ Velocistas } & \multirow{2}{*}{5} & $x$ & 32,2 & 3 & 2,2 & 9,4 & 63,1 \\
\hline & & $d p$ & 20,88 & 4,00 & 2,56 & 3,26 & 39,99 \\
\hline \multirow{2}{*}{ Lançadoras } & \multirow{2}{*}{4} & $x$ & 69,75 & 33,00 & 1,75 & 10,50 & 99,25 \\
\hline & & $\mathrm{dp}$ & 21,04 & 10,25 & 1,92 & 2,29 & 34,47 \\
\hline \multirow{2}{*}{ Fundistas } & \multirow{2}{*}{5} & $\mathrm{x}$ & 30,80 & 21,40 & 3,20 & 9,20 & 71,40 \\
\hline & & $d p$ & 25,59 & 15,87 & 3,71 & 3,54 & 37,58 \\
\hline \multicolumn{8}{|c|}{ Homens } \\
\hline \multirow{2}{*}{ Velocistas } & \multirow{2}{*}{6} & $\mathrm{x}$ & 18,50 & 3,17 & 1,0 & 10,50 & 72,83 \\
\hline & & $d p$ & 24,0 & 4,6 & 0,8 & 0,8 & 13,9 \\
\hline \multirow{2}{*}{ Fundistas } & \multirow{2}{*}{9} & $x$ & 6 & 2,43 & 1,57 & 11,29 & 93,93 \\
\hline & & $\mathrm{dp}$ & 2,8 & 1,6 & 3,5 & 4,4 & 47,3 \\
\hline
\end{tabular}


Tabela 8 - Média (x) e desvio padrão (dp) do rendimento alcançado nos testes de aptidão física das mulheres e homens integrantes do CIED de Atletismo da I Região

\begin{tabular}{|c|c|c|c|c|c|c|c|}
\hline & $50 \mathrm{~m}$ & $\mathrm{v}\left(\mathrm{m} \cdot \mathrm{s}^{-1}\right)$ & Drible & Sargent & Potência & $6 \mathrm{~min}$ & $\mathrm{VO}_{2 \text { max }}$ \\
\hline \multicolumn{8}{|c|}{ mulheres } \\
\hline \multicolumn{8}{|c|}{ velocistas } \\
\hline$x$ & 8,02 & 6,23 & 11,17 & 0,33 & 119,09 & 1244,00 & 42,27 \\
\hline$d p$ & 0,44 & 0,79 & 0,55 & 0,08 & 7,73 & 27,92 & 0,55 \\
\hline \multicolumn{8}{|c|}{ lançadoras } \\
\hline$x$ & 8,84 & 5,65 & 12,08 & 0,33 & 138,51 & 1049,00 & 38,45 \\
\hline$d p$ & 0,57 & 0,57 & 0,29 & 0,04 & 40,42 & 61,96 & 1,22 \\
\hline \multicolumn{8}{|c|}{ fundistas } \\
\hline$x$ & 8,25 & 6,06 & 11,55 & 0,33 & 119,09 & 1375,00 & 44,83 \\
\hline$d p$ & 0,31 & 0,4 & 0,46 & 0,02 & 15,29 & 68,67 & 1,35 \\
\hline \multicolumn{8}{|c|}{ homens } \\
\hline \multicolumn{8}{|c|}{ velocistas } \\
\hline$x$ & 6,84 & 7,30 & 10,09 & 40,50 & 128,40 & 1364,00 & 44,63 \\
\hline$d p$ & 0,31 & 0,81 & 0,46 & 4,87 & 30,37 & 69,61 & 1,37 \\
\hline \multicolumn{8}{|c|}{ fundistas } \\
\hline$x$ & 7,89 & 6,33 & 11,36 & 38,00 & 121,50 & 1510,00 & 47,51 \\
\hline$d p$ & 0,45 & 0,16 & 0,16 & 6,70 & 16,13 & 206,70 & 4,06 \\
\hline
\end{tabular}

Tabela 9 - Índices de correlação entre os desenhos dermatoglíficos e a especialidade atlética dos homens integrantes de CIED da I Região

\begin{tabular}{lcccccc}
\hline & \multicolumn{3}{c}{ homens velocistas } & \multicolumn{3}{c}{ homens fundistas } \\
\cline { 2 - 7 } & $50 \mathrm{~m}$ & potência & $\mathrm{VO}_{2}$ & $50 \mathrm{~m}$ & potência & $\mathrm{VO}_{2}$ \\
\hline $\mathrm{L}$ & 0,95 & 0,92 & 0,87 & 0,75 & 0,85 & 0,89 \\
$\mathrm{~A}$ & 0,79 & 0,82 & 0,67 & 0,39 & 0,36 & 0,98 \\
$\mathrm{~W}$ & 0,23 & 0,56 & 0,83 & 0,61 & 0,39 & 0,85 \\
$\mathrm{D} 10$ & 0,75 & 0,71 & 0,89 & 0,84 & 0,91 & 0,90 \\
SQTL & 0,97 & 0,89 & 0,61 & 0,54 & 0,54 & 0,60 \\
\hline
\end{tabular}

do que as mulheres e, portanto, ao comparar ambos os grupos de velocistas (mulheres e homens), os homens apresentam 58,76\% de SQTL maior que as mulheres.

Não obstante, ao fazer a comparação entre fundistas homens e mulheres, se apresenta um fato muito especial, já que neste caso são as mulheres que apresentam na sua mão direita $5,5 \%$ de maior intensidade de desenho que os homens. No entanto, na mão esquerda, são os homens que superam as mulheres por uma percentagem de $5,07 \%$, uma diferença não significativa com $p \geq 0,05$.

Outro fato, que também se repete em ambos os sexos da amostra, é a superioridade que apresentam as médias SQTL dos dedos da mão direita sobre seus homólogos da mão esquerda.

\section{Aptidão física}

Tal como se planejou na metodologia, a aptidão física foi medida e avaliada, mediante a aplicação dos instrumentos de medição específicos, para as qualidades físicas de velocidade de deslocamento, força explosiva e resistência aeróbica, nos quais se obtiveram os resultados apresentados na Tabela 6. Nela se pode observar que, em relação à velocidade de deslocamento, estes se enquadram dentro do esperado, segundo as especialidades estudadas. Isto é, são os velocistas, tanto mulheres como homens, que apresentam a maior velocidade, com um alcance de 6,23m.s ${ }^{-1}$ e 7,3 m.s. $\mathrm{s}^{-1}$ nas mulheres e homens, respectivamente. Portanto, é evidente que os sujeitos mais lento são as mulheres lançadoras, com uma velocidade de $5,65 \mathrm{~m} \cdot \mathrm{s}^{-1}$, e os homens fundistas, com uma velocidade de $6,33 \mathrm{~m} \cdot \mathrm{s}^{-1}$.

Quanto à avaliação da potência extensora de joelho (teste de Sargent), pode-se manifestar que homens velocistas são os que apresentam o maior desenvolvimento da potência. A despeito das mulheres da amostra, os valores médios mais altos registrados nesta qualidade alcançaram $128,40 \pm 30,37 \mathrm{kgm} \cdot \mathrm{s}^{-1}$, enquanto nas mu-

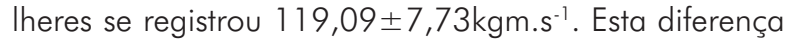
é proporcionada pela maior capacidade do salto apresentado pelos homens velocistas que, além disso, apresentam uma massa (peso) média superior. No entanto, são as mulheres lançadoras as que apresentam a maior potência extensora de joelho, ao alcançar uma média de $138,51 \pm 40,42 \mathrm{kgm} . \mathrm{s}^{-1}$, o que pode ser atribuído à alto 
Tabela 10 - Índices de correlação entre os desenhos dermatoglíficos e a especialidade atlética das mulheres integrantes de CIED da I Região

\begin{tabular}{|c|c|c|c|c|}
\hline \multicolumn{5}{|c|}{ velocistas } \\
\hline & $50 \mathrm{~m}$ & Sargent & potência & $\mathrm{VO}_{2}$ \\
\hline $\mathrm{L}$ & 0,92 & 0,94 & 0,93 & 0,97 \\
\hline W & 0,78 & 0,73 & 0,69 & 0,47 \\
\hline A & 0,68 & 0,73 & 0,60 & 0,41 \\
\hline D10 & 0,83 & 0,91 & 0,87 & 0,53 \\
\hline SQTL & 0,77 & 0,88 & 0,68 & 0,84 \\
\hline \multicolumn{5}{|c|}{ lançadoras } \\
\hline & $50 \mathrm{~m}$ & Sargent & potência & $\mathrm{VO}_{2}$ \\
\hline $\mathrm{L}$ & 0,86 & 0,89 & 0,97 & 0,79 \\
\hline W & 0,72 & 0,65 & 0,65 & 0,67 \\
\hline A & $-0,72$ & 0,95 & 0,84 & 0,96 \\
\hline D10 & $-0,83$ & 0,87 & 0,96 & 0,86 \\
\hline SQTL & $-0,60$ & 0,73 & 0,06 & 0,87 \\
\hline \multicolumn{5}{|c|}{ fundistas } \\
\hline & $50 m$ & Sargent & potência & $\mathrm{VO}_{2}$ \\
\hline $\mathrm{L}$ & $-0,97$ & 0,94 & 0,62 & 0,90 \\
\hline W & $-0,95$ & 0,86 & 0,86 & 0,84 \\
\hline A & $-0,79$ & 0,67 & 0,85 & 0,35 \\
\hline D10 & $-0,96$ & 0,95 & 0,82 & 0,85 \\
\hline SQTL & $-0,92$ & 0,94 & 0,84 & 0,87 \\
\hline
\end{tabular}

média do peso corporal, além de ser um grupo muito heterogêneo devido a alto desvio padrão do grupo.

No caso da resistência aeróbica, representada pelo $\mathrm{VO}_{2 \text { máx }}$ alcançado mediante a aplicação do teste de $6 \mathrm{~min}$ de corrida contínua, podemos dizer que são os homens fundistas os que apresentam o maior consumo de oxigênio, apesar de seus valores se encontrarem baixos em relação aos parâmetros internacionais atribuído a esta variável, o que se pode atribuir à diferença de idades e níveis de rendimento que apresentou a amostra escolhida.

\section{Correlações}

Como se nota na Tabela 9, os homens velocistas apresentaram correlações mais elevadas na potência e no $\mathrm{VO}_{2 \text { máx }}$ em relação às características dermatoglíficas de L e SQTL. No entanto, apresentam uma correlação baixa entre o desenho dermatoglífico "W" e a potência e a velocidade. Não assim nos fundistas que na sua correlação apresentam boa afinidade do $\mathrm{VO}_{2 \text { máx }}$ com os desenhos W - A e a intensidade sumária dos desenhos (D10).

Na Tabela 10 podemos observar que as mulheres velocistas apresentam uma correlação positiva muito forte, de 0,92 a 0,97, entre o desenho dermatoglífico " $L$ " e a velocidade, a potência e o $\mathrm{VO}_{2 \text { máx }}$. Da mesma forma, se nota uma boa correlação entre a intensidade sumária D10 com a velocidade, a potência extensora de pernas. No entanto, sua correlação com o VO ${ }_{2 \text { máx }}$ é baixa. Por sua vez, as lançadoras apresentam uma elevada correlação do desenho $L$ em relação à velocidade e à potência, $e$ aceitável com $\circ \mathrm{VO}_{2 \text { máx }}$. Além disso, se pode dizer que há uma baixa relação entre o desenho $\mathrm{W}$, as variáveis de velocidade, potência e $\mathrm{VO}_{2 \text { máx }}$

No caso das fundistas se nota uma boa relação em $80 \%$ dos desenhos e as variáveis contrastadas em estudo.

\section{DISCUSSÃO}

Com relação às hipóteses de estudo, pode-se manifestar que:

- Nas mulheres, há uma marcada tendência à apresentação de desenho tipo $L$ e A, se associando estes, muito fortemente, à velocidade e à força. De fato, suas médias, em todos os níveis, são superiores aos obtidos pelo desenho tipo W.

- Nos homens, se percebe uma leve diferença a favor da apresentação de desenhos tipo L e A. No entanto, há um equilíbrio comparado com a aparição dos desenhos tipo W.

- Os desenhos datiloscópicos dos dedos das mãos, tanto nas mulheres como nos homens, explicam uma elevada relação entre as qualidades físicas de velocidade e resistência com a combinação dos desenhos L-W e A. Por outro lado, as lançadoras apresentam grande afinidade dos desenhos L-A e D10 com a força de potência.

\section{REFERÊNCIAS}

1. Filin VP, Volkov VM. Seleção de talentos nos desportos. Londrina: Midiograf; 1998.

2. Fernandes Filho J. Impressões Dermatoglíficas - Marcas Genéticas no Seleção dos tipos de Esportes e Lutas (a exemplo de desportistas do Brasil) [tese]. Moscou: Instituto de Investigação Científica de Cultura Física Esportes da Rússia; 1997.

3. Bulgakova N. Seleção e preparação de jovens nadadores. Educação Física e Esporte. Moscou; 1978

4. Nikitiuk B, Filipov V. Complexo dermatoglífico que acompanha o alto desenvolvimento das qualidades motoras. Método biológico de gêmeos, médico e esporte. Vinitza; 1984

5. Abramova TF, Nikitina TM. Tipo das impressões digitais nos atletas de elite de diferentes esportes. Atualidades médicas e antropológicas nos esportes. Moscou; 1990.

6. Abramova TF, Nikitina TM, Ozolin NN. Possibilidades de utilização das Impressões dermatoglíficas na seleção desportiva. Teoria e prática da cultura física. Moscou; 1995.

7. Abramova TF, Nikitina TM, Ozolin NN. Impressões Dermatoglíficas nos atletas de alta qualificação de diferentes esportes. Problemas morfológicos nos esportes. Moscou: Volvograd; 1992.

8. Abramova TF, Ozolin NN, Martirosov EG. Impressões digitais como índices de prognóstico na iniciação da orientação esportiva. Problemas morfológicos na seleção esportiva. Moscou; 1989 